\title{
Research Paper \\ Evaluation of Factor Structure of the Persian Scale of Coping With Carver Shortened Stress
}

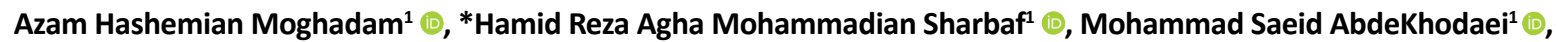 \\ Hossein Kareshki ${ }^{1}$ (1)
}

1. Department of Psychology, Faculty of Education and Psychology, Ferdowsi University of Mashhad, Mashhad, Iran.

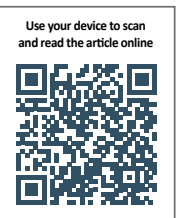

Cttation: Hashemian Moghadam A, AghaMohammadian Sharbaf HR, AbdeKhodaei MS, Kareshki H. [Evaluation of Factor Structure of the Persian Scale of Coping With Carver Shortened Stress (Persian)]. Journal of Arak University of Medical Sciences (JAMS). 2020; 23(4):472-487. https://doi.org/10.32598/JAMS.23.4.4488.2

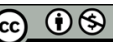

Article Info:

Received: 16 Feb 2020 Accepted: 17 Jun 2020 Available Online: 01 Oct 2020

Keywords:

Coping skills, Exploratory factor analysis, Confirmatory factor analysis evaluating, Extended abstract

\section{A B STRACT}

Background and Aim Coping with stress is one of the most important research areas in health psychology. Researching in this regard requires a tool with strong psychometric properties and validation in Iranian culture. The purpose of this study was to investigate the most commonly used short-scale factor structure, the Carver's Brief Cope.

Methods \& Materials This study was a cross-sectional and methodological study of test type. The statistical population consisted of all students studying at Birjand University in 1977-98. Sampling was available by the method. The condition for entry into the study was the experience of a stressor for at least 6 months. After receiving a medium or high score on the perceived stress level, 629 students completed the 28-question form. Data was analyzed using SPSS V. 15 and laser software V. 8.8. Exploratory factor analysis was performed to determine the factor structure of the questionnaire. A confirmatory factor analysis was used to confirming the factors. Reliability was established through retesting and internal consistency. Ethical Considerations This study is part of a PhD. thesis, registered (Code: IR.UM.REC. 3/50099) at Ferdowsi University of Mashhad. Before entering the study, an informed consent was obtained from the subjects. Results After confirming the face and content validity quantitatively and qualitatively, the exploratory factor analysis results after varimax rotation showed $55.139 \%$ of the total variance explained by the first eight factors with a higher than one specific value. However, in confirmatory factor analysis, the first two factors, 4 and 7, were omitted due to insufficient power to explain the present variables. Finally, the goodness-of-fit indices of the RMFIA, PFI, GFI, IFI, IFI, CFI, AGFI confirmed the fit of the six-factor structure with the data. Reliability of the instrument was also confirmed by internal consistency $(\alpha=0.73)$ and test-retest reliability $(r=0.59)$

Conclusion The highly abbreviated 6-item form of Carver's Brief Cope showed good validity and reliability in the student sample and can be used in health psychology studies.

\section{Extended Abstract}

\section{Introduction}

oping with stress is one of the most impor-

tant areas of research in health psychol- ogy. Carver, Skier, and Winthrop have distinguished a wide range of coping strategies and raised the need to design a comprehensive and transparent tool in the form of a theoretical framework [18]. They first developed the long form of the Countermeasures Preference Questionnaire [18, 19].

\section{* Corresponding Author:}

Hamid Reza AghaMohammadian Sharbaf, PhD.

Address: Department of Psychology, Faculty of Education and Psychology, Ferdowsi University of Mashhad, Mashhad, Iran.

Tel: +98 (51) 38805865

E-mail: aghamohammadian@ferdowsi.um.ac.ir 
However, Carver presented 28 questions and 14 factors due to the shortened form's length $[13,20]$. The 14-factor structure has not been confirmed in a significant number of researches and cultural contexts, and using exploratory factor analysis, other models of this tool including 11-factor model [27], 9-factor [quoted], 7-factor [24], 8 factors [25], and 4 factors [17] are presented in the research background. This questionnaire has not yet been localized in Iran; therefore, this study aimed to present a version with psychometrically powerful features in the Iranian student population for the first time by examining the factor structure and psychometric properties of the abbreviated version of the preference of coping practices.

\section{Materials and Methods}

This research was a cross-sectional and testing type methodological study. The statistical population was all students of Birjand University in 2018-2019. The sampling method was available. The condition for entering the study was to experience stress for at least the last 6 months. After earning an average or high score on perceived stress, 629 students completed the 28-item form. Data were analyzed using SPSS version 15 and LISREL software version 8.8. Since the 14-factor structure was not run, exploratory factor analysis was performed to determine the questionnaire's functional design. The tools included historical information, two long forms, and a shortened form of the Brief-COPE Questionnaire.

Confirmatory factor analysis was used to confirm the factors. AGFI, RMSEA, PNFI, GFI, IFI, CFI indexes were evaluated. Reliability was performed by internal consistency coefficients and retesting by 129 people two weeks apart. Assessing the convergent validity, the correlation coefficients between the six factors of the abbreviated form questionnaire and the five factors of the Iranian version of the long form of confrontational preference were calculated by 47 students who answered two questionnaires simultaneously.

\section{Results}

Formal validity was confirmed based on the Wilde method and the principles of reverse translation and content validity by calculating the two indexes of content validity ratio and content validity index [31]. Confirmatory factor analysis was used to evaluate the construct validity. Due to the non-implementation of the model, exploratory factor analysis was used to determine this questionnaire's factor structure. As shown in Table 1, the Kaiser-Meier-Alkin sampling index (KMO) was equal to 0.745 , and the Bartlett sphericity test with a rate of 3584.130 and a degree of freedom of 378 was significant at the level of 0.0001 .
As shown in Table 2, the exploratory factor analysis results after varimax rotation showed that the first 8 factors explained $55.139 \%$ of the total variance with a specific value higher than one. As shown in Table 3, GFI, IFI, CFI, AGFI indexes had values less than 0.90, which indicates the unacceptability of the indexes and the incompatibility of the 8-factor model with the data (Table 3). To modify the model, factor load values and t-statistic were investigated. In the first-order factor structure, the t-values in the four markers of factor 7 were less than 1.96, which was omitted. In the second-order factor structure, the value of the t-statistic related to latent variable 4 with a total factor of 0.01 was less than 1.96 , so factor 4 did not have enough power to explain the general factor was omitted. Finally, RMSEA, PNFI, GFI, IFI, CFI, AGFI goodness-of-fit indicators confirmed the fit of the 6-factor structure with the data. The instrument's reliability was also confirmed by internal consistency $(\alpha=0.73)$ and retest $(r=0.59)$.

All retest correlation coefficients were significant for the factors 1, 2, 3, 5, 6 and 8, which were: 0.68, 0.60, 0.60, 0.70, 0.60 and 0.56 , respectively, at the level of 0.000 . Cronbach's alpha for the whole instrument was 0.73 , which indicates the acceptable internal consistency of the test questions.

\section{Discussion and Conclusion}

In the present study, items related to planning factors, active coping, positive reformatting, and acceptance (in the main form of 14 factors) were loaded on factor 1 . This factor was consistent with the positive coping factor in Mizaki's 7-factor structure [24] and was called problem-focused coping. The items related to the search for instrumental support and receiving emotional support were loaded on factor 2, following the support-oriented confrontation of the latent 4-factor structure [27], which was called support-focused coping. Factors 3, 5, and 6 , both of which relate to self-distraction, religion, and denial, respectively, were loaded according to the original 14-factor form [20] and therefore retained under the same names. Finally, factor 8 , which is the item related to the two factors of emotional discharge and selfblame, was following the axis of escape factor in the structure of 4 latent factors [27] and was named negative emotion-focused coping.

The highest internal consistency was related to factors 1,2 , and 6, respectively. Of course, factors 1 and 2 can show both the high internal consistency of the items and the diversity of things influenced by them. All factors had a positive and significant correlation with the total score. Religion had the highest correlation with the general factor, which in the Iranian religious community 
Table 2. Rotated matrix of the structure of the coping questionnaire

\begin{tabular}{|c|c|c|c|c|c|c|c|c|}
\hline Items & First Factor & Second Factor & Third Factor & Fourth Factor & Fifth Factor & Sixth Factor & Seventh Factor & Eighth Factor \\
\hline 14 & 0.69 & & & & & & & \\
\hline 2 & 0.68 & & & & & & & \\
\hline 2 & 0.66 & & & & & & & \\
\hline 7 & 0.54 & & & & & & & \\
\hline 17 & 0.47 & & & & & & & \\
\hline 20 & 0.40 & & & & & & & \\
\hline 12 & 0.37 & & & & & & & \\
\hline 10 & & 0.79 & & & & & & \\
\hline 23 & & 0.77 & & & & & & \\
\hline 5 & & 0.64 & & & & & & \\
\hline 15 & & 0.61 & & & & & & \\
\hline 19 & & & 0.65 & & & & & \\
\hline 1 & & & 0.52 & & & & & \\
\hline 9 & & & 0.57 & & & & & \\
\hline 6 & & & 0.37 & & & & & \\
\hline 4 & & & & 0.82 & & & & \\
\hline 11 & & & & 0.81 & & & & \\
\hline 27 & & & & & 0.85 & & & \\
\hline 22 & & & & & 0.82 & & & \\
\hline 8 & & & & & & 0.79 & & \\
\hline 3 & & & & & & 0.74 & & \\
\hline 16 & & & & & & & 0.66 & \\
\hline 28 & & & & & & & 0.59 & \\
\hline 18 & & & & & & & 0.47 & \\
\hline 26 & & & & & & & & 0.76 \\
\hline 21 & & & & & & & & 0.56 \\
\hline 24 & & & & & & & & 0.43 \\
\hline 13 & & & & & & & & 0.36 \\
\hline
\end{tabular}

Table 3. Fitness indexes of the abbreviated form of the Counter-Preference Action Questionnaire (Brief-COPE)

\begin{tabular}{|c|c|c|c|c|c|c|c|c|c|}
\hline \multirow{2}{*}{$\begin{array}{c}\text { Fitness Index Type } \\
\text { Fitness indices and their ac- } \\
\text { ceptable values }\end{array}$} & \multicolumn{5}{|c|}{ Absolute } & \multicolumn{2}{|c|}{ Relative } & \multicolumn{2}{|c|}{ Summary } \\
\hline & $\chi^{2}$ & $\mathrm{df}$ & $\chi^{2} / d f$ & RMSEA $<0.08$ & $\mathrm{GFI}>0.90$ & $\mathrm{IFI}>0.90$ & $\mathrm{CFI}>0.90$ & AGFI $>0.90$ & PNFI $>0.50$ \\
\hline $\begin{array}{l}\text { Structural pattern of 6-factor } \\
\text { model }\end{array}$ & $787.94^{* *}$ & 194 & 4.6 & 0.075 & 0.98 & 0.91 & 0.91 & 0.95 & 0.53 \\
\hline $\begin{array}{c}\text { Structural pattern of } 8 \text { factor } \\
\text { model }\end{array}$ & $1296.20 * *$ & 322 & 4.02 & 0.069 & 0.87 & 0.83 & 0.83 & 0.84 & 0.67 \\
\hline
\end{tabular}


Table 1. Special value, percentage of variance, and Compression percentage of 8 factors

\begin{tabular}{cccc}
\hline & & & (\%) \\
\cline { 3 - 4 } Factors & Special Value & Variance & Compression \\
\cline { 3 - 4 } & & 15.22 & 15.22 \\
\hline 2 & 2.26 & 8.62 & 23.85 \\
\hline 3 & 1.95 & 6.99 & 30.84 \\
\hline 4 & 1.60 & 5.73 & 36.57 \\
\hline 5 & 1.48 & 5.31 & 41.89 \\
7 & 1.39 & 4.99 & 46.89 \\
\hline 8 & 1.27 & 4.55 & 51.44 \\
\hline
\end{tabular}

shows the highest preference for coping with stressors in the Iranian student community. Factors 5, 1, 2, 3, 6, and 7 had the highest correlation coefficient, respectively. Therefore, the most important coping skills that students prefer in the face of perceived stress were religion orientation, problem-focused coping, support-focused coping, distraction, denial, and emotion-focused coping, respectively.

\section{Ethical Considerations}

\section{Compliance with ethical guidelines}

This study has been approved by Ferdowsi University of Mashhad (Code: IR.UM.REC. 3/50099). All ethical principles are considered in this article. The participants were informed of the purpose of the research and its implementation stages.

\section{Funding}

This study is part of a $\mathrm{PhD}$. dissertation of first author, Department of Psychology, Faculty of Education and Psychology, Ferdowsi University of Mashhad.

\section{Authors' contributions}

Conceptualization: Hamid Reza AghaMohammadian Sharbaf, Azam Hashemian Moghadam; Research and sampling method: Azam Hashemian Moghadam; Data analysis: Hossein Kareshki, and Azam Hashemian Moghadam; Drafting: Azam Hashemian Moghadam, Hamid Reza AghaMohammadian Sharbaf; Editing and finalizing: Ha- mid Reza AghaMohammadian Sharbaf, Mohammad Saeid AbdeKhodaei, and Hossein Kareshki.

\section{Conflicts of interest}

The authors have no conflict of interest to declare.

\section{Acknowledgements}

The authors would like to express their gratitude to the staff of Omid and Imam Reza hospitals, as well as the patients admitted to the hospitals for their collaboration. 
This Page Intentionally Left Blank 


\section{ارزيابى ساختار عاملى مقياس فارسى مقابله با استرس كوتاهشده كارور در دانشجويان دانشغًاه بيرجن}

اعظم هاشميان مقدم' (1) 'حميدرضا آقامحمديان شعرباف' (1)، محمدسعيد عبد خدايى' (i)، حسين كارشكى

1. كروه روانشناسي، دانشكده علوم تربيتى و روانشناسى، دانشكاه فردوسى مشهد، مشهبه ايران.

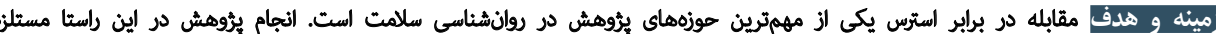

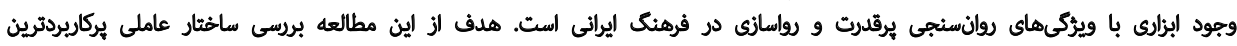
مقياس ثلاصهشده يعنى فرم كوثاه مقابله كارود بودئ.

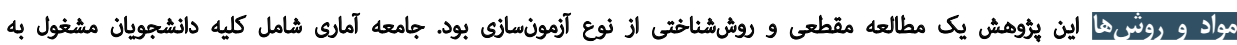

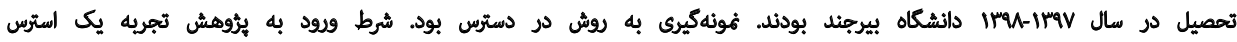

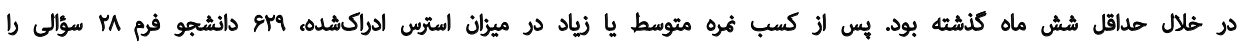

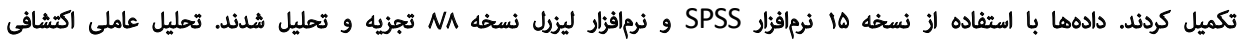

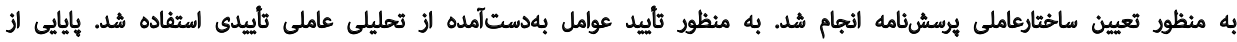

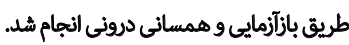

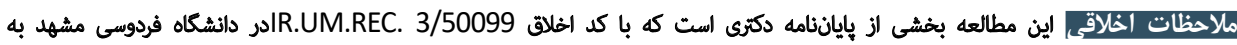

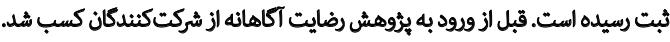

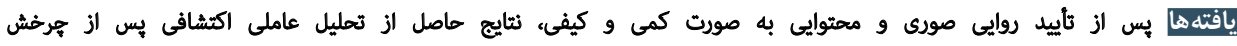

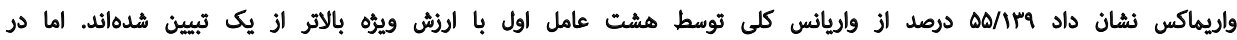

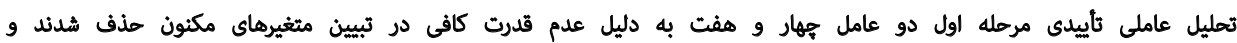

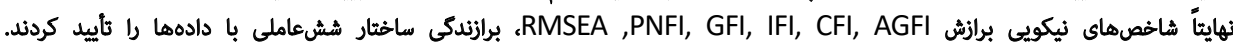

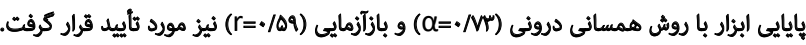

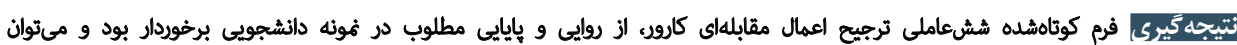

مقابله جستوجو وتبيين سعاختار مقابله برداشتهاند و از طرفيى

مقدهd

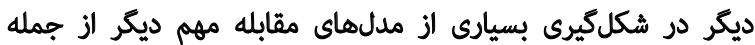

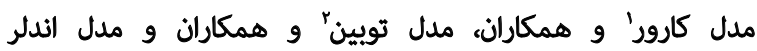

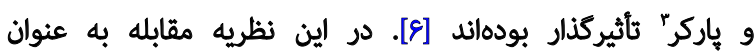

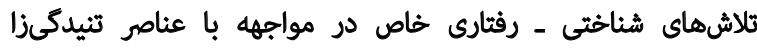

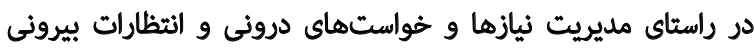

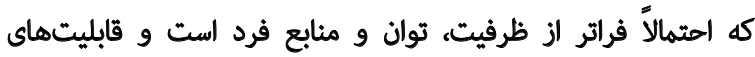

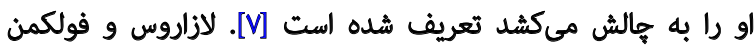
مقابله را به دو طبقه متمركز بر مسئله و متمركز بر هيجان تقديم لقديم

1. Carver

2. Tobin

3. Endler \& Parker
مقابله مفهومى بسيار كسترده، با بيشينه تاريخى طولانى و وانه

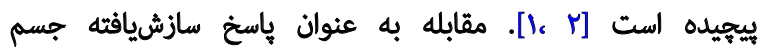

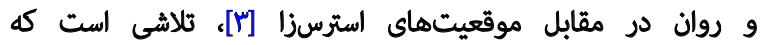

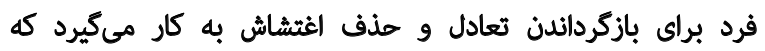

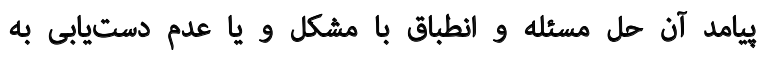

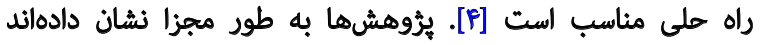

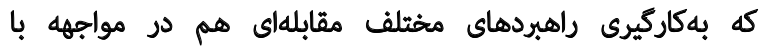

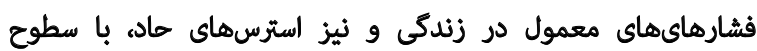

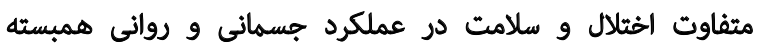

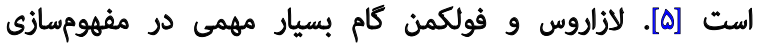

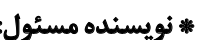
حميدرخا آقامحمديان شعرباف مشئف

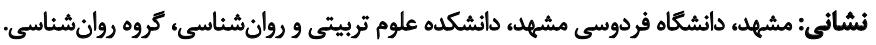
تلفن:

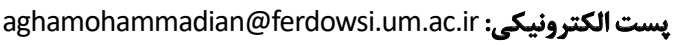


بنابراين كارور فرم كوتاهشده اين مقياس را با حفظ هواردارده عامل

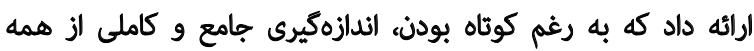

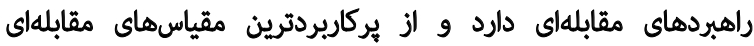

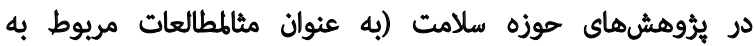

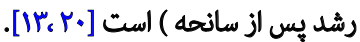
اين يرسشامه يكى از سه يرسشنامه الصلى اندازمكيرى

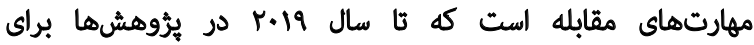

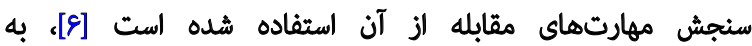

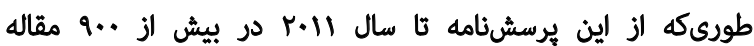

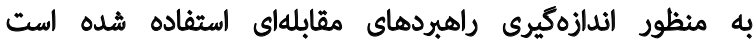
[اr]]. اين ابزار در بسيارى از كشورهاى ارويايى، آمريكاى شمالى

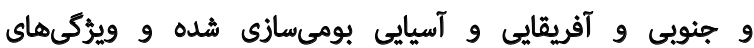

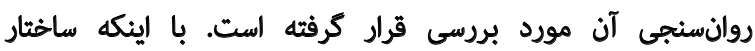

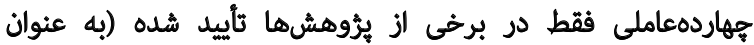

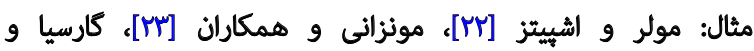

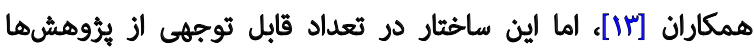

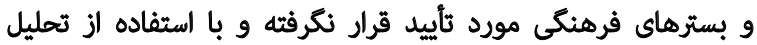

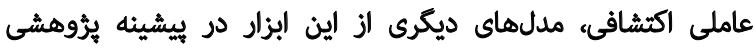

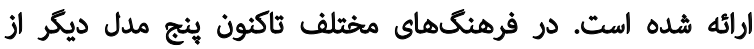

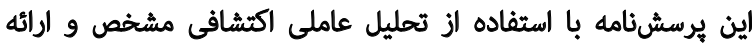

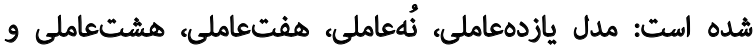

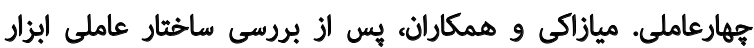

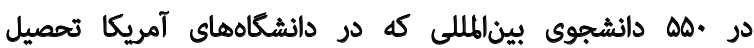

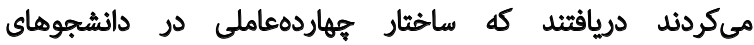

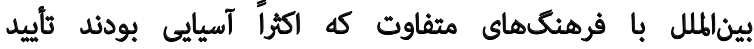

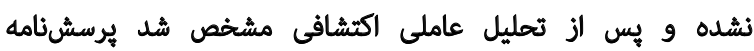

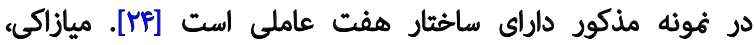

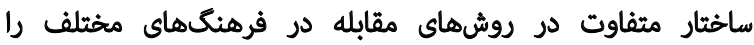

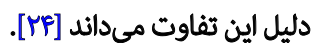

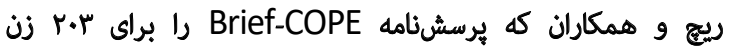

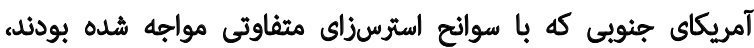

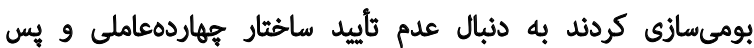

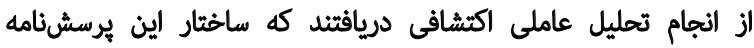

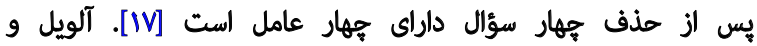

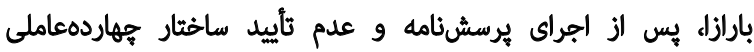

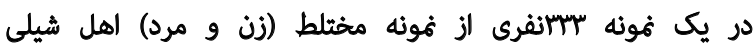

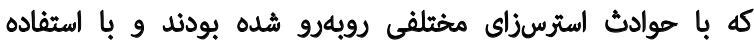

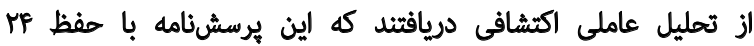

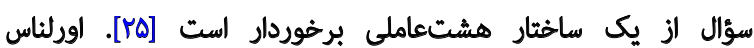

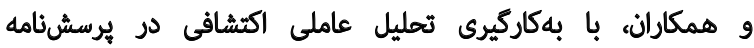

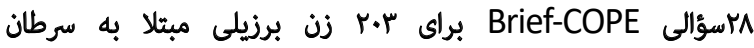

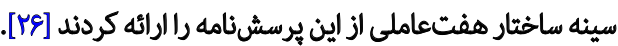

8. Cope Operations Preference Enquary (Brief-COPE)
و بر اساس همين طبقهبندى يرسشنامه روشهاى مقابله" خود

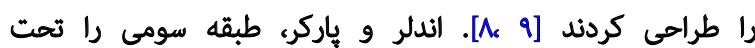

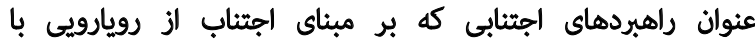

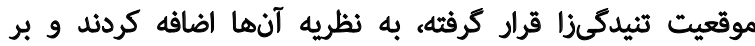

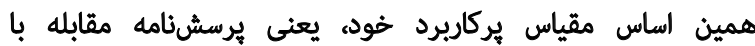

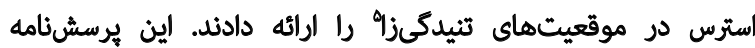

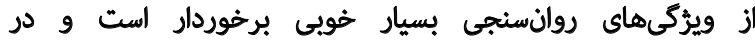

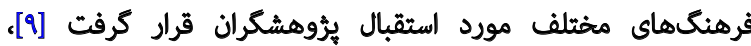

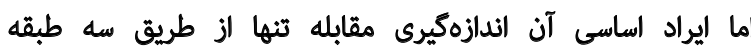

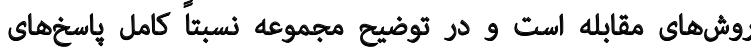

مقابلهاى ناتوان است [ [ [1].

لازاروس بعدها اذذعان داشت تمايز بين مقابله مثمركز بر مسئله و

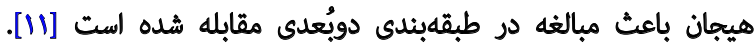

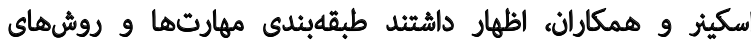

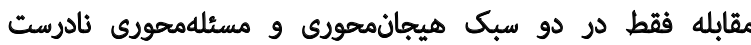

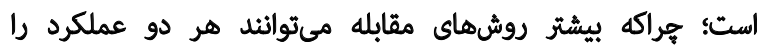

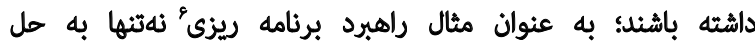

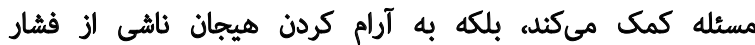

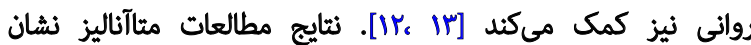

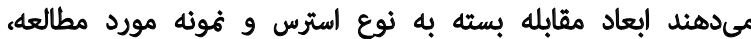

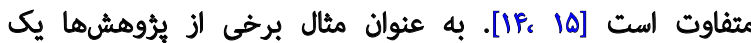

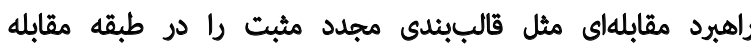

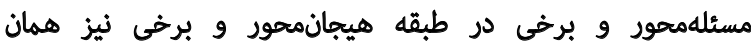

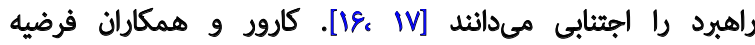

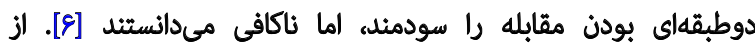

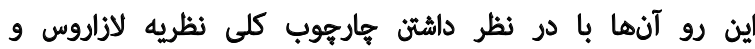

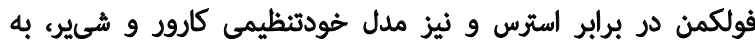

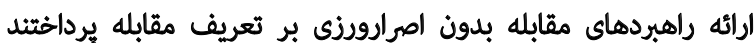

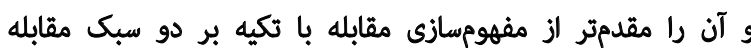

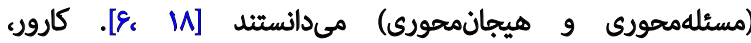
اسكير و وينتراب با توجه به تأكيدى كه بر تفاوتهاي فردئ فردي

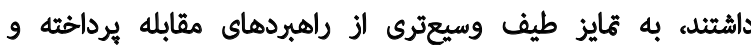

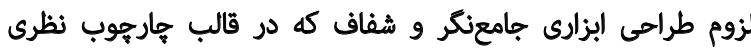

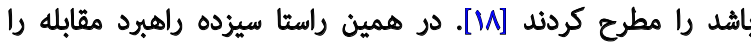

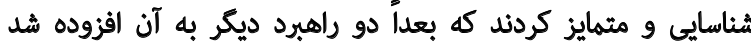

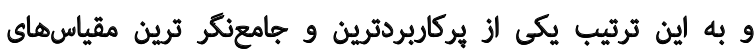

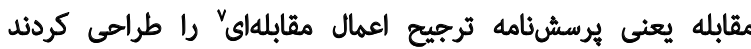

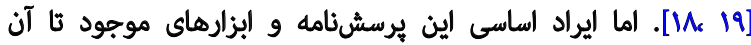

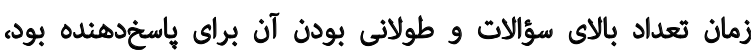

4. Ways of Copping Questionnaire (WCQ)

5. Coping Inventory for Stressful Situation(CISS)

6. Plannig

7. Cope Operations Preference Enquary (COPE) 


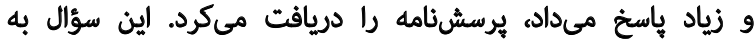

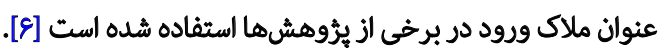

كواثل منظور بررسى روايى صورى، نسخه اصلى يرسشنامه

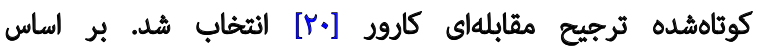

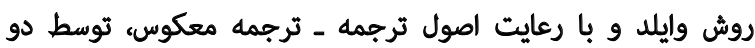

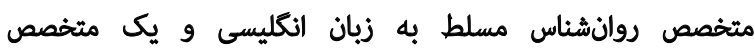
مسلط برسشنامه از انكليسى به فارسى و و بالعكس ترجماسه. مقايسه، اصلاح و نهايى شد [•"r].

روايى محتوايى از طريق محاسبه دو شاخص نسبت روايى

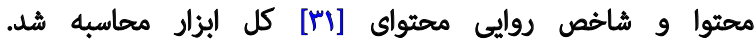

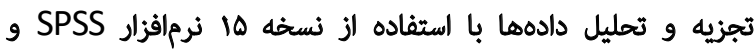

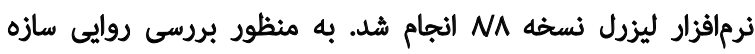

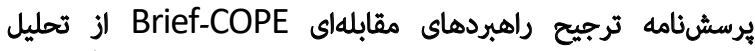

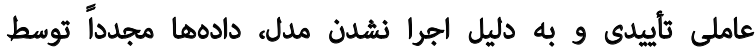

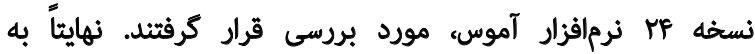

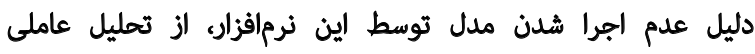

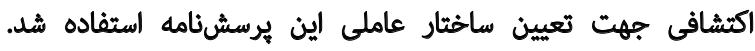

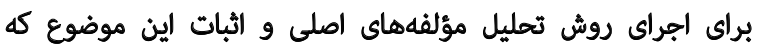

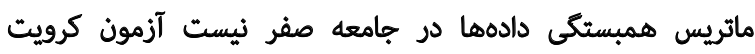

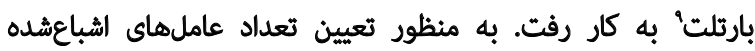

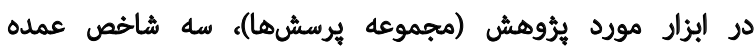

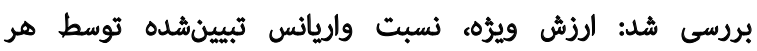

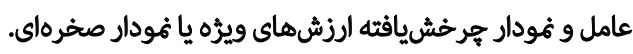

يس الز تعيين عوامل به منظور تأييد عوامل بهدستآمده از

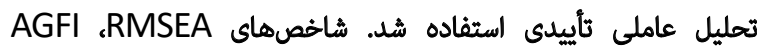

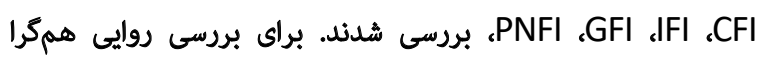

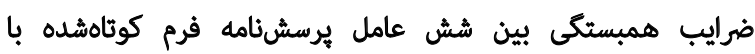

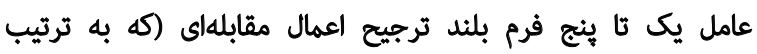

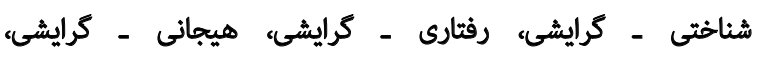

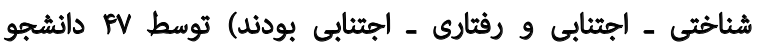

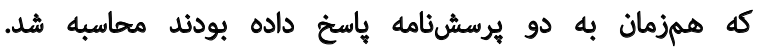

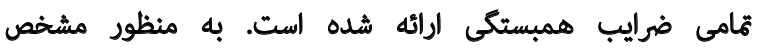

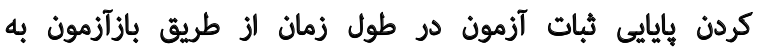

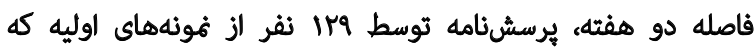

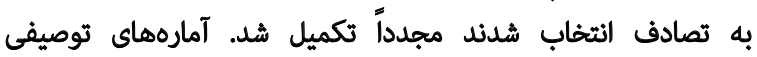

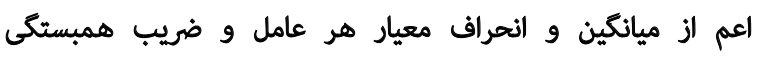
ييرسون بين هر عامل با هـره كل نيز الرائه شيده است.

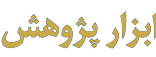

فرم رضايت آكاهانه و اطلاعات ثاريخهِالى شامل سن، جنس، سطح تحصيلات و محل سكونت بود.

9. Bartlett's Test of Sphericity (BT)
كنول و همكاران كه اين يُرسشنامه را در يك نمونه آيمانى

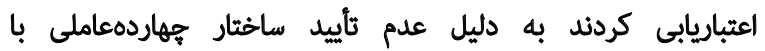

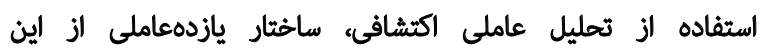

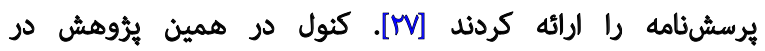

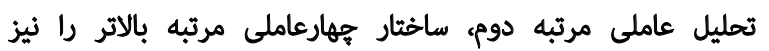

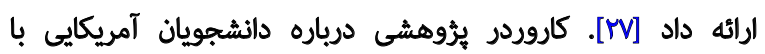

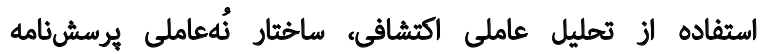

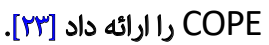

يس از بررسى تمامى بإيكاههاى داخلى و خارجى از جمله

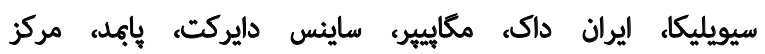

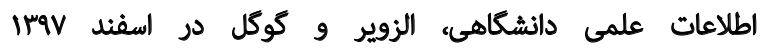

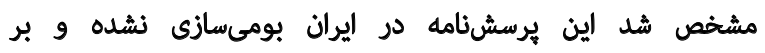

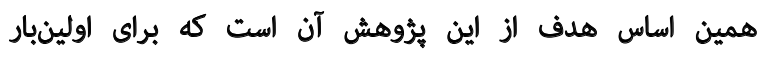

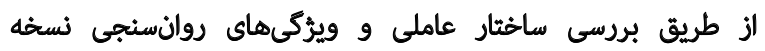

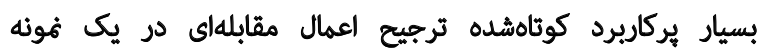

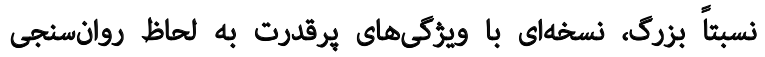
رادر جمعيت دانشجويى ايرانى اراثه كند.

مواد و روشها

مطالعه حاضر يك مطالعه روششناغتى از نوع آزمونسازى بود

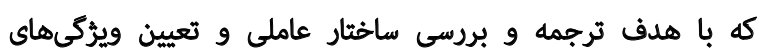

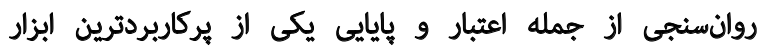

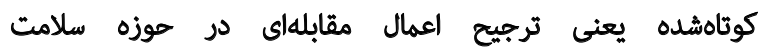

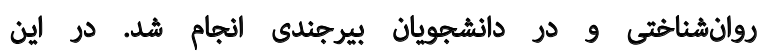

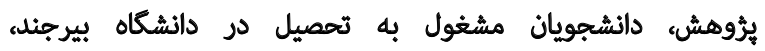

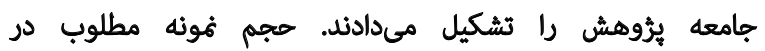

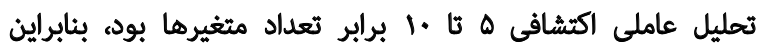

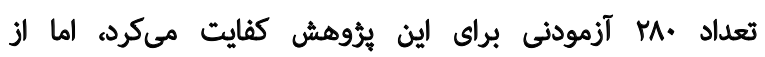

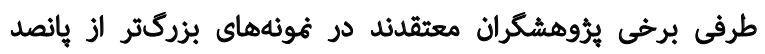

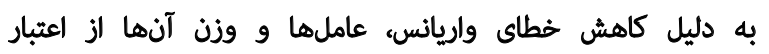

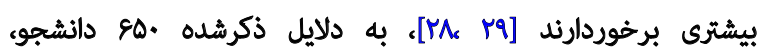

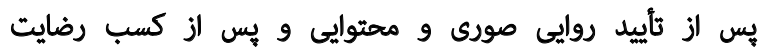

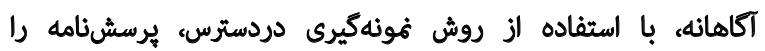

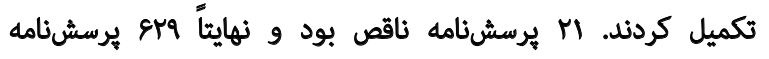

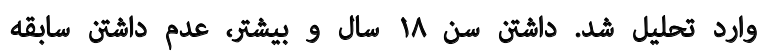

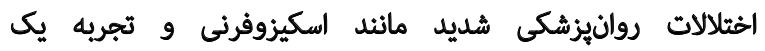

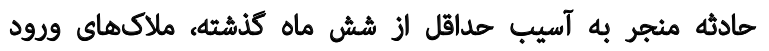

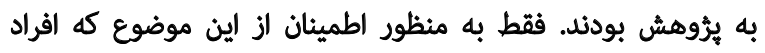

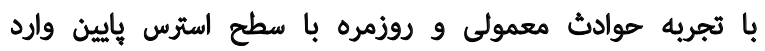

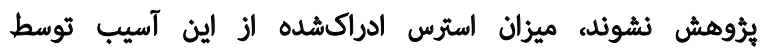

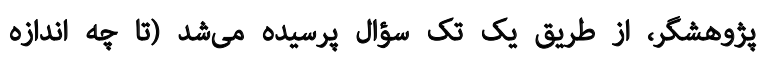

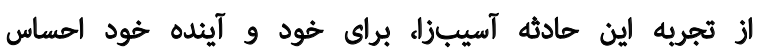

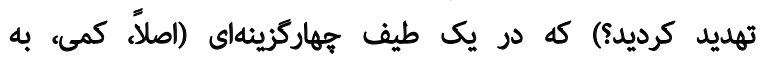

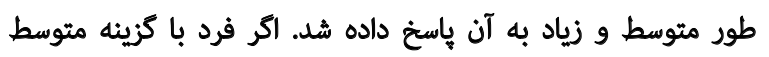




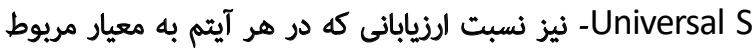

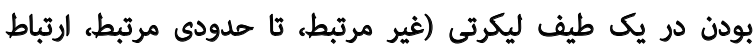

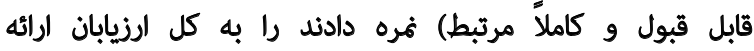

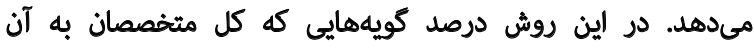

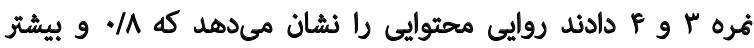

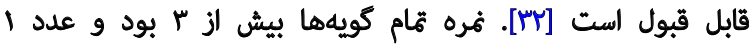
براي اين شاخص نشاندهنده روايى محتوايى مناسب است. روايى سازه: مقدار شاخص نمونهكيرى كيسر ـ ماير ـ الكين"

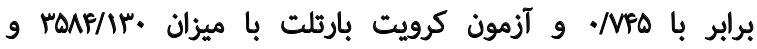

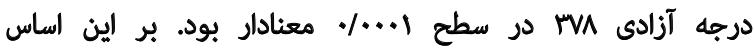

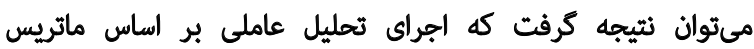

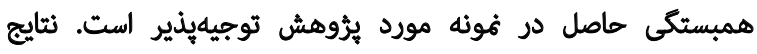

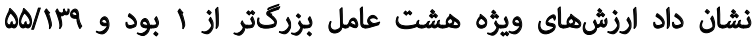

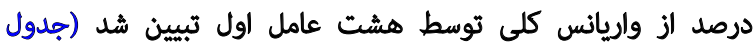

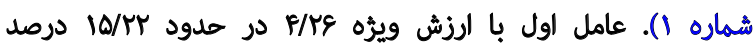

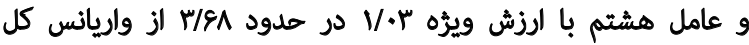

را تبيين مىكنتد (جدول شماره 1).

تمودار صخرهاى نشان داد سهم عامل اول مهمتر و متهايز

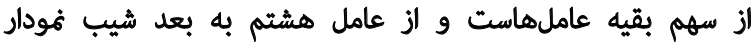
تقريبا هموار شده است. طبق نتايج تحليل ساختارى اكتشافى در

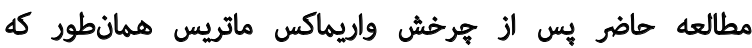

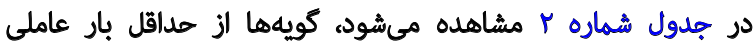

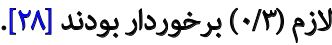

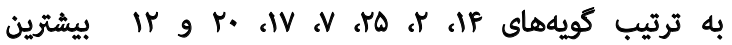

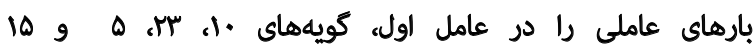

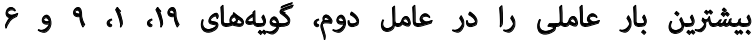

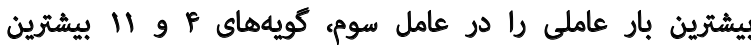

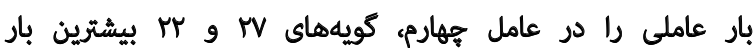

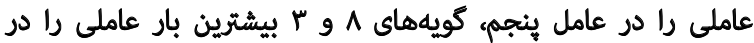

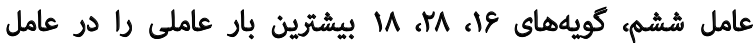

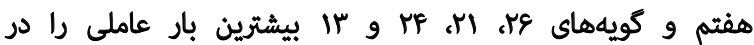

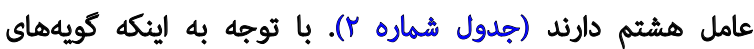

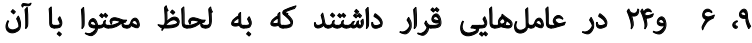

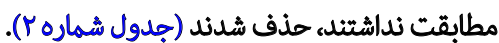

يس از شناسايى ساختار متغيرها، به منظور تأييد و بررسى

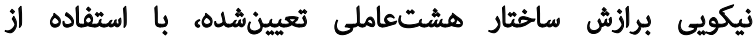

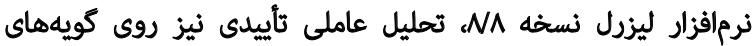

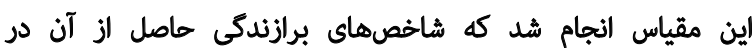

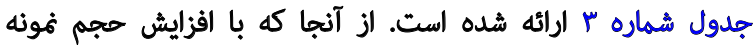

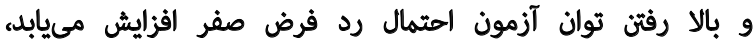

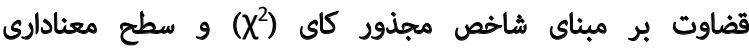

12. Kaiser-Mayer-Olkin (KMO)
يرسشنامه شصتسؤالى ترجيح اعمال مقابلهائ": اين ابزال

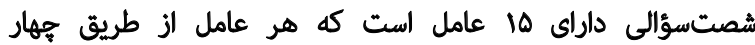

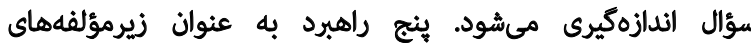

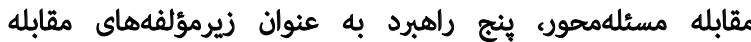

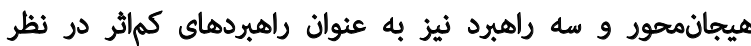

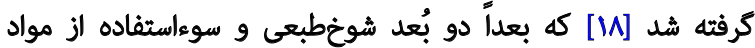

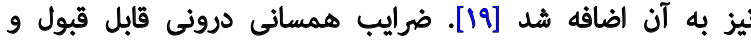

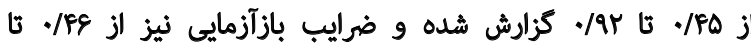

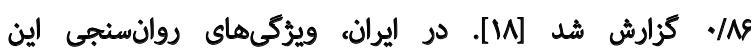

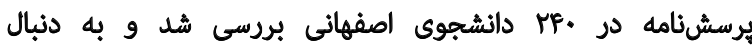

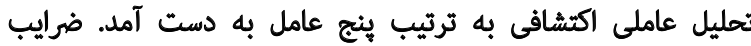

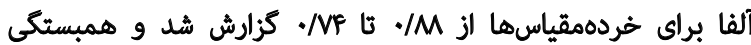

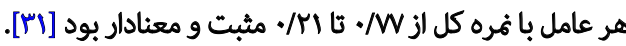
فرم كوتاهشده يرسشنامه ترجيح اعمال مقابلهاي: كارور اين

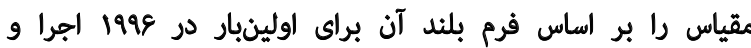

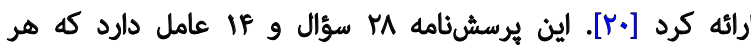

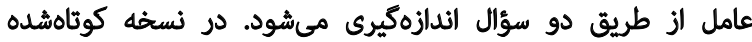

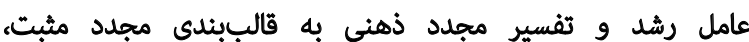

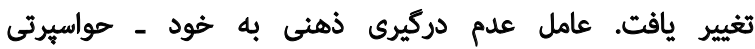

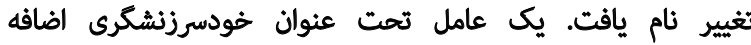

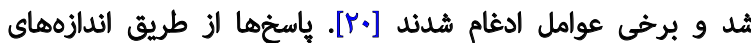

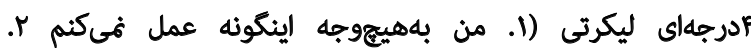

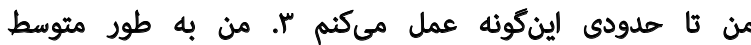

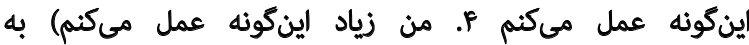

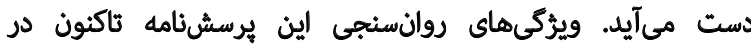

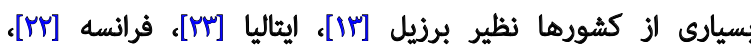

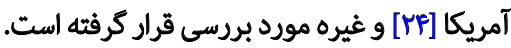

ياقتهها

در قسمث آمار توصيفى مربوط به نمونه مورد مطالعه ميانكين

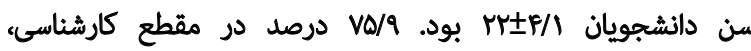

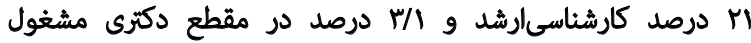

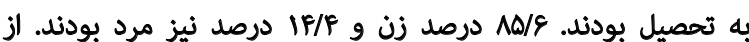
اين تعداد A درصد بومى و Ar درصد غيربومى بودند.

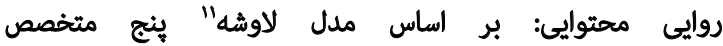

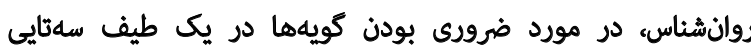

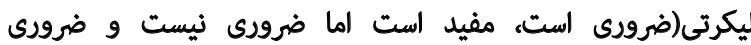

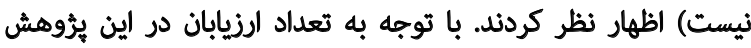

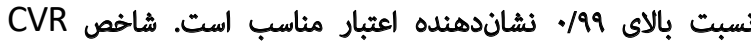

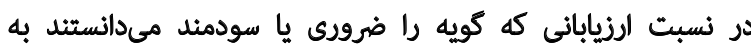

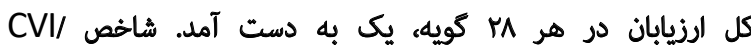

\section{COPE}

11. Lawshe 
جدول ا. ارزش ويزٔه، درصد تبيين واريانس و درصد تراكمى عوامل هشت كانه

\begin{tabular}{|c|c|c|c|}
\hline دوصد تراكمى & درصد واريانس & ارزش ويره & عوامل \\
\hline $10 / \pi r$ & $10 / \pi Y$ & P/RE & 1 \\
\hline rr/AA & Nar & $r / F i$ & $r$ \\
\hline Y./AF & $8 / 99$ & $1 / 90$ & $r$ \\
\hline re/ar & $\Delta / N^{\omega}$ & $1 / 8$. & f \\
\hline F)/199 & ( ו & $1 / \uparrow A$ & $\Delta$ \\
\hline PE/Aq & $4 / 99$ & $1 / 49$ & 8 \\
\hline (1)/ $/ 4$ & $P / \Delta \Delta$ & $1 / m$ & $\gamma$ \\
\hline$\Delta \Delta / / P q$ & r/FA & $Y / \cdot r$ & $A$ \\
\hline
\end{tabular}

بايايى مقياس: ميانكين و النحراف معيار (داخل يرانتن) عوامل

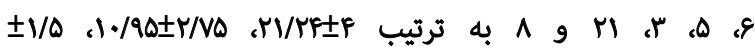

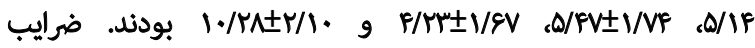

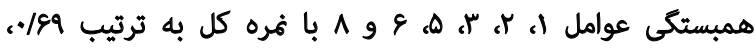
19T

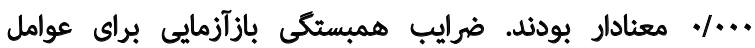

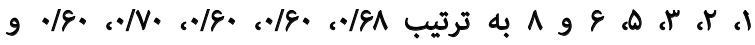

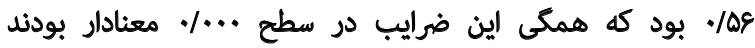
كه نشاندهنده ثبات بسيار خوب اين ابزار در طول زمان است. ضريب آلفاي كرونباخ به منظور تعيين توافق درونى ابزار

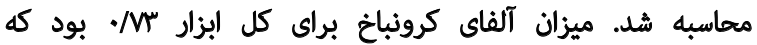

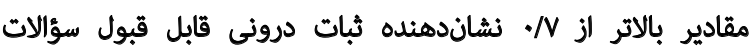

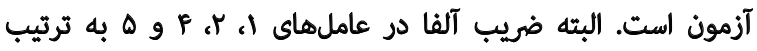

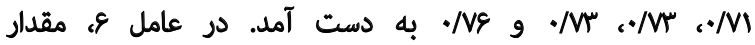

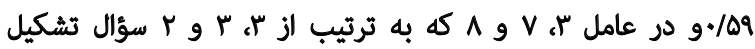

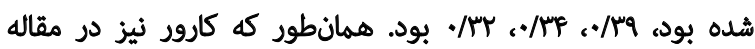

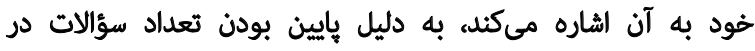

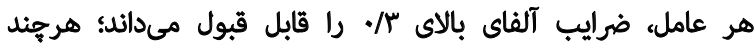

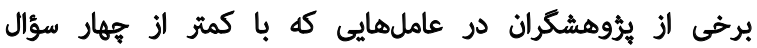

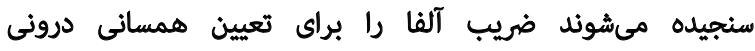

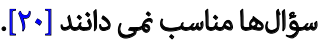

\section{ث}

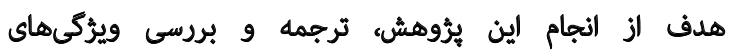

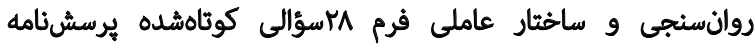

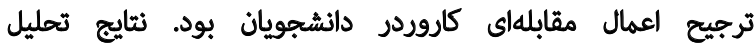

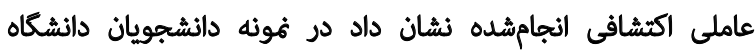

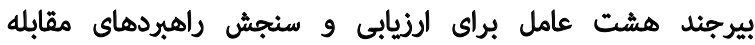

آن كمراه كنيده است [TH]، بنابراين در اين يخؤهش به منظور

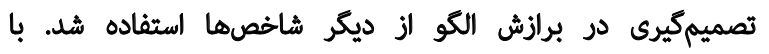

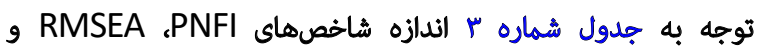

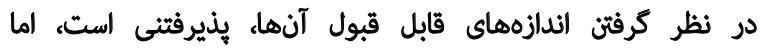

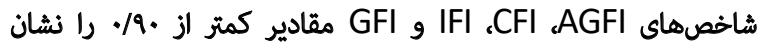
مىدهند كه نشاندهنده عدم مقبوليت شاخصها و و بنابراين

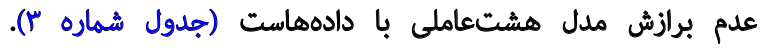

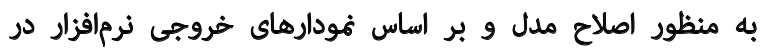
ابتدا مقادير بار عاملى و آماره تى متغيرهاى مشاهدهيذير (كويهها)

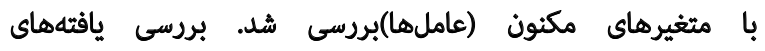
حاصل از الجراى آزمون تى (در سطح معنادارى 1.

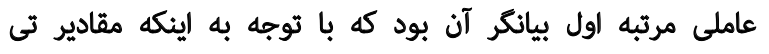

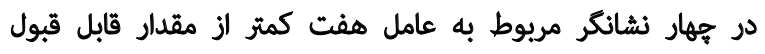

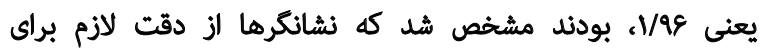

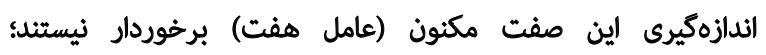

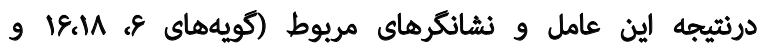
(MA) به آن حذف شدند.

از طرفى در برربسى ساختار عاملى مرتبه دوم به دليل اينكه مقدار

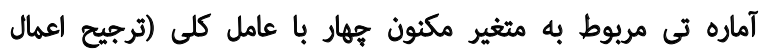

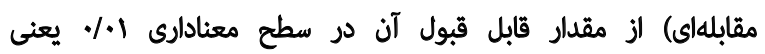

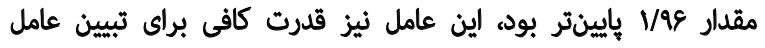

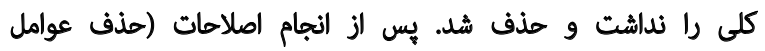

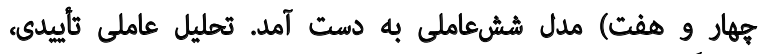

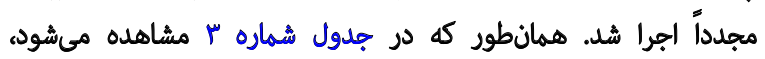

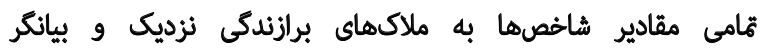

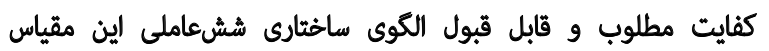

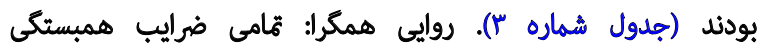

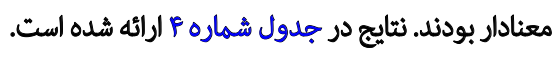


جدول r. ماتريس جرخش دادهده ساختار يرسشئامه ترجيح اعمال مقابلهاي

\begin{tabular}{|c|c|c|c|c|c|c|c|c|}
\hline عامل هشتم & عامل هفتم & عامل ششم & عامل ينجم & عامل خهارم & عامل سوم & عامل دوم & عامل اول & كويه \\
\hline & & & & & & & .189 & if \\
\hline & & & & & & &. $\mid 8 A$ & $r$ \\
\hline & & & & & & & .188 & ro \\
\hline & & & & & & & $\cdot(\Delta)^{P}$ & $v$ \\
\hline & & & & & & & - INV & IV \\
\hline & & & & & & &.$/ 4$ & $r$. \\
\hline & & & & & & & $\cdot / m$ & ir \\
\hline & & & & & & . 199 & & 1. \\
\hline & & & & & & $\cdot M$ & & MT \\
\hline & & & & & & .189 & & $\Delta$ \\
\hline & & & & & & .181 & & 10 \\
\hline & & & & & .180 & & & 19 \\
\hline & & & & & . $/ \Delta T$ & & & 1 \\
\hline & & & & & $\cdot / \Delta V$ & & & 9 \\
\hline & & & & & $\cdot / M V$ & & & 8 \\
\hline & & & & - IAT & & & & $f$ \\
\hline & & & & $\cdot(A)$ & & & & 11 \\
\hline & & & - INA & & & & & Tr \\
\hline & & & - /AT & & & & & Mr \\
\hline & & - $N 9$ & & & & & & $\Lambda$ \\
\hline & & $\cdot M F$ & & & & & & $r$ \\
\hline & . I\&8 & & & & & & & is \\
\hline &.$/ 199$ & & & & & & & M \\
\hline &.$/ F r$ & & & & & & & $u$ \\
\hline$\cdot / V G$ & & & & & & & & re \\
\hline.$/ \Delta S$ & & & & & & & & rI \\
\hline T/R. & & & & & & & & $M F$ \\
\hline . & & & & & & & & ir \\
\hline
\end{tabular}


جدول r. شاخصهاى برازندكى فرم كوتاهشه يرسشنامه ترجيح اعمال مقابلهاى (Brief-COPE)

\begin{tabular}{|c|c|c|c|c|c|c|c|c|c|}
\hline \multicolumn{2}{|c|}{ موجز } & \multicolumn{2}{|c|}{ نسبي } & \multicolumn{5}{|c|}{ مطلق } & \multirow{2}{*}{ 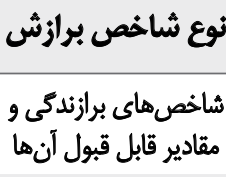 } \\
\hline $\begin{array}{l}\text { PNFI } \\
>* / \Delta .\end{array}$ & $\begin{array}{l}\text { AGFI } \\
>* / 9 .\end{array}$ & $\mathrm{CFI}>+/ 9$. & $\begin{array}{c}\text { IFI } \\
>+/ 9 .\end{array}$ & $\mathrm{GFI}>.19$. & $\begin{array}{c}\text { RMSEA } \\
<\bullet / \bullet \wedge\end{array}$ & $\chi^{2} / \mathrm{df}$ & $d f$ & $\chi^{2}$ & \\
\hline.$/ \Delta r$ &.$/ 90$ & .191 & .191 &.$/ 44$ & H.VA & p/s & $19 f^{\circ}$ & VAV/qF $=*$ & شش الكوى ساختارى مدلى \\
\hline $.18 V$ & - MAP & $\cdot / A r$ & . & - /AV & $1.8 q$ & $H / r$ & Mrr & $\mid r q \& / T . \ldots$ & الكوى ساختارى هدل \\
\hline
\end{tabular}

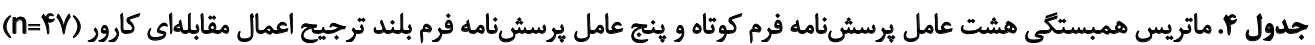

\begin{tabular}{|c|c|c|c|c|c|}
\hline كل & شناختى ـ-اجتنابى & هيجانى ـ ـكرايشى & رفتارى ـ-كرايشى & شناختى - كرايشى & عامل \\
\hline & & & & $.18 Q^{\circ *}$ & مقابله مثبت مسئلهمحور \\
\hline & & & 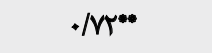 & & مقابله حمايتمحور \\
\hline & &.$|8|^{* *}$ & & & خودجواسيرتى \\
\hline & $.18 \theta^{2 *}$ & & & & كرايش به مذهب \\
\hline & &.$/ 4 \Lambda^{* * *}$ & & & انكار \\
\hline & & & 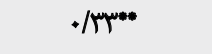 & & مقابله منفى هيجاندحور \\
\hline.$M n^{e+*}$ & & & & & كل ابززار \\
\hline
\end{tabular}

مقابله، يذيرش مشكل و رويارويى فُعال و عدم انكار است، باركيرى

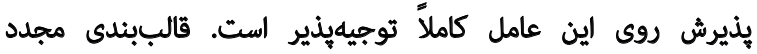

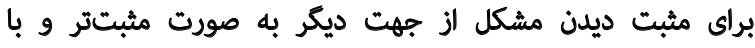

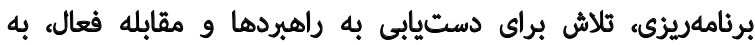

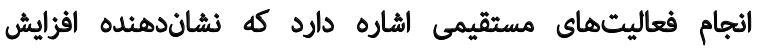

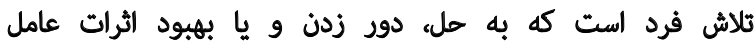

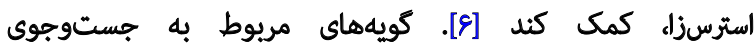
حمايت ابزارى (دريافت كمك يا راهنمايى و مشاوره از ديكران

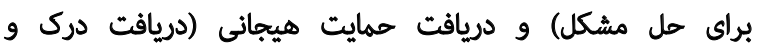

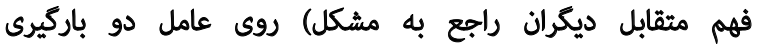

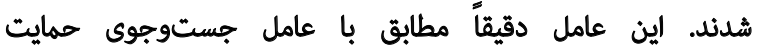

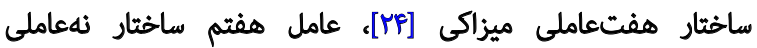

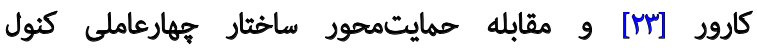

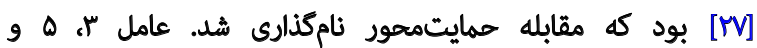

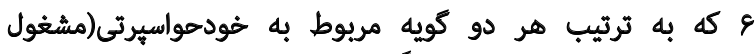

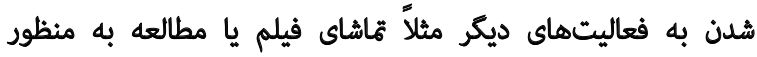

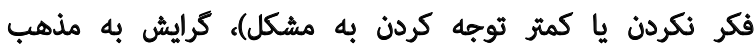
(دستيابى به آرامش از طريق عقايد مذهبى و معنويت درد مواجهه
در برابر استرس ادراكشده مناسب بود. اما يس از انجام تحليل

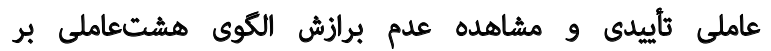

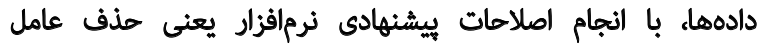

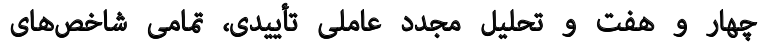

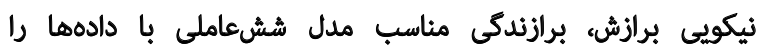

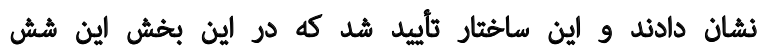
عامل تبيين شدند.

در يروهش حاضر كويههاى مربوط به عاملهاى برنامهريزى،

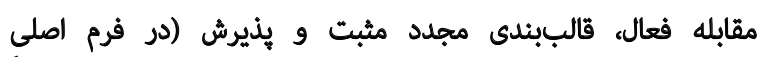

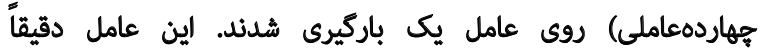

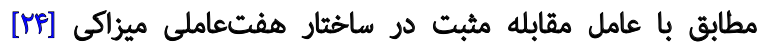

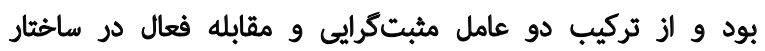

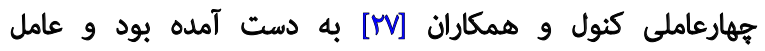

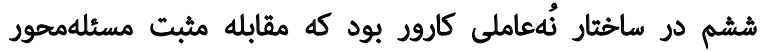
نامكذارى شدم. همانطور كه ميزاكى [MF] نيز در يُروهش خود به آن آن اشاره

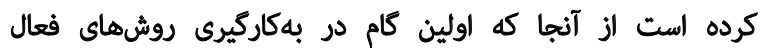




\section{ماحظات اخلاقى - احق

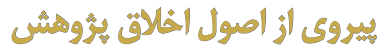

اين مطالعه با كد اخلاق IR.UM.REC. 3/50099 در دانشكاه

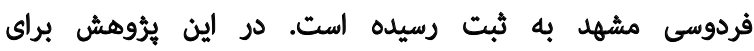

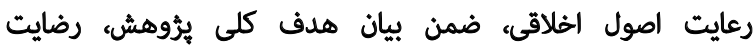

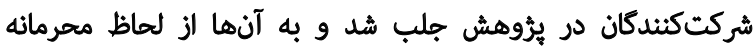
ماندن اطلاعات نيز اطمينان داده شد.

$$
\text { Ito }
$$

اين يُروهش از رساله دكتى نويسنده اول، در كروه روانشناسى دانشكده علوم تربيتى، دانشكاه فردوسى مشهد استخراج شدره

\section{مشاركت نويسندكّان}

مفهومسازى: حميدرضا آقامحمديان و اعظم هاشميان مقدم؛

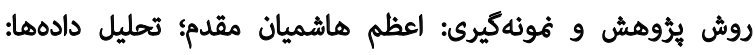

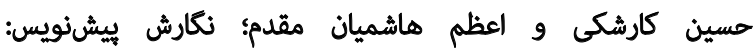

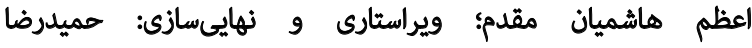
آقامحمديان شعرباف، محمد سعيد عبدخدايى و حسين كارشكى.

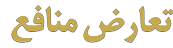

بنابر الظهار نويسندكان اين مقاله هيجكونه تعارض مثافعى

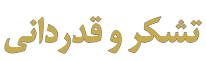

نويسندكان الز تمامى برسنل و بيماران بيمارستانهاى اميد و

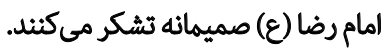

با مشكل) و انكار (رد كردن و عدم هذيرش واقعيت وجودى بودي

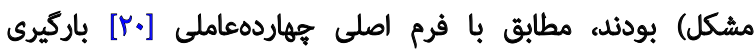

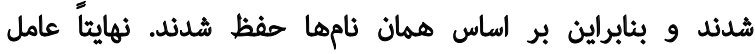
هشت كه كويههاي مريوط به دو عامل تخليه هيجاني نانى (بيان و ابراز

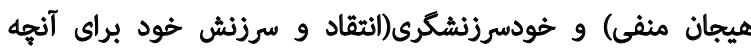
اتفاق افتاده)، بر آن باركيرى شدنده مطابق

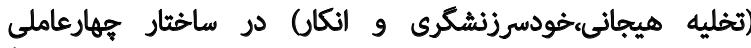

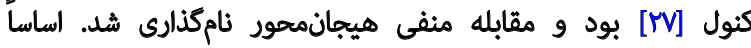

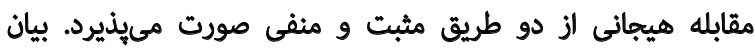

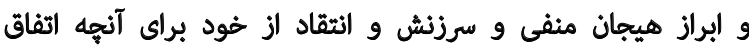

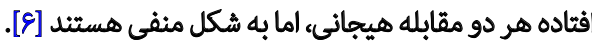

در آخر ضريب آلفاى كل نشاندهنده همسانى درونى قابل قبول مقياس بود. بيشترين همسانى درونى به به ترتيب مرئ مريوط

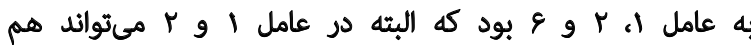

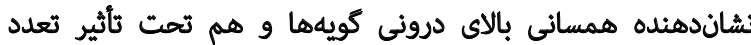

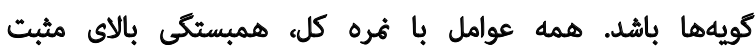

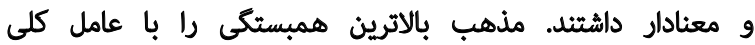

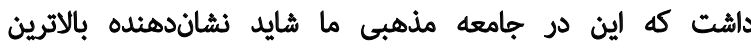

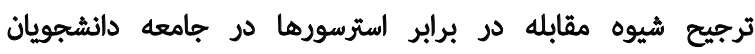

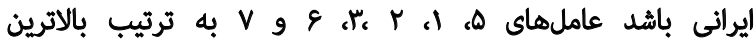

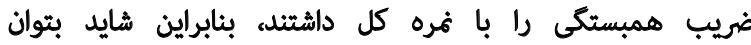

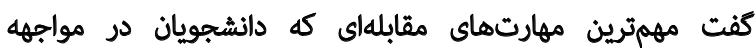

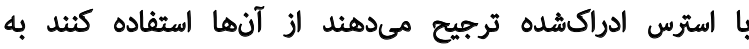
ترثيب كرايش به مذهب، مقابله مسئلهمحور، مقابله حمايتمحورو، حواسيرتى، النكار و مقابله هيجانمحور بودند.

$$
\text { نتيجه كئيرى }
$$

نتايج اين يُروهش روايى و ثايايى مطلوب فرم كوتاششده ششعاملى ترجيح مهارتهاي مقابله (مقابله مثبت مسئلهمحورو،

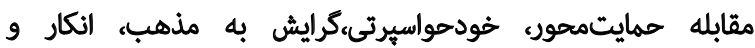

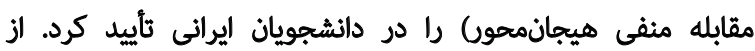

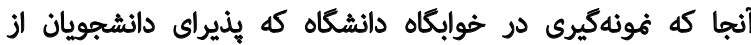

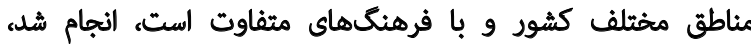

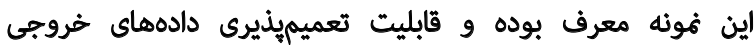

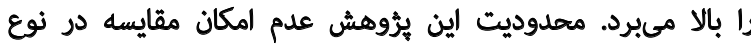

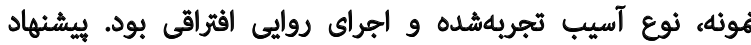

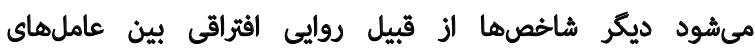

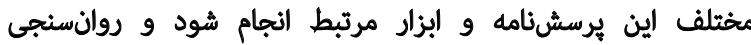

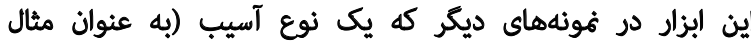
سرطان) را تجريه كردهاند مورد بررسى و مقايسه قرار كيرند. 


\section{References}

[1] Smith MM, Sherry SB, Rnic K, Saklofske DH, Enns M, Gralnick T. Are perfectionism dimensions vulnerability factors for depressive symptoms after controlling for neuroticism? A meta-analysis of 10 longitudinal studies. Eur J Pers. 2016; 30(2):201-12. [DOI: 10.1002/per.2053]

[2] Liang SY, Liu HC, Lu YY, Wu SF, Chien CH, Tsay SL. The influence of resilience on the coping strategies in patients with primary brain tumors. Asian Nurs Res. 2020; 14(1):50-5. [DOI: 10.1016/j.anr.2020.01.005] [PMID]

[3] Brasileiro SV, Orsini MRCA, Cavalcante JA, Bartholomeu D, Montie JM, Costa PSS, et al. Controversies regarding the psychometric properties of the brief COPE: The case of the brazilian-portugueseversion "COPE breve". PloS One. 2016; 11(3):e0152233. [DOI:10.1371/journal. pone.0152233] [PMID] [PMCID]

[4] Compas BE, Jaser SS, Bettis AH, Watson KH, Gruhn MA, Dunbar JP, et al. Coping, emotion regulation, and psychopathology in childhood and adolescence: A meta-analysis and narrative review. Psychol Bull Adv. 2017; 143(9):939-91. [DOI:10.1037/bul0000110] [PMID] [PMCID]

[5] Meng X, D’Arcy C. Coping strategies and distress reduction in psychological well-being? A structural equation modelling analysis using a national population sample. Epidemiol Psychiatr Sci. 2016; 25(4):370-83. [DOI:10.1017/S2045796015000505] [PMID] [PMCID]

[6] Stanisławski K. The Coping circumplex model: An integrative model of the structure of coping with stress. Front Psychol. 2019; 10:694-716. [DOI:10.3389/fpsyg.2019.00694] [PMID] [PMCID]

[7] Folkman S, Lazarus RS, Dunkel-Schetter C, DeLongis A, Gruen RJ. Dynamics of a stressful encounter: Cognitive appraisal, coping, and encounter outcomes. J Pers Soc Psychol. 1986; 50(5):992-1003. [DOI:10.1037/0022-3514.50.5.992] [PMID]

[8] Seiler A, Sood AK, Jenewein J, Fagundes CP. Can stress promote the pathophysiology of brain metastases? A critical review of biobehavioral mechanisms. Brain Behav Immun. 2020; 87:860-80. [DOI:10.1016/j. bbi.2019.12.013] [PMID]

[9] Boysan M. Psychometric properties of the turkish version of the coping inventory for stressful situations. Noro Psikiyatr Ars. 2012; 49(3):196202. [DOI:10.4274/npa.y6192]

[10] Schwarzer R, Schwarzer C. A critical survey of coping instruments. In: Zeidner M, Endler NS, editors. Handbook of coping. New York, NY: Wiley; 1996. http://userpage.fu-berlin.de/gesund/publicat/copchap6.htm

[11] Lazarus RS. The role of coping in the emotions and how coping changes over the life course. In: Maletesta-Magni C, McFadden SH, editors. handbook of emotion,adult development, and aging. New York, NY: Academic Press; 1996. [DOI:10.1016/B978-012464995-8/50017-0]

[12] Skinner EA, Edge K, Altman J, Sherwood H. Searching for the structure of coping: A review and critique of category systems for classifying ways of coping. Psychol Bull. 2003; 129(2):216-69. [DOI:10.1037/00332909.129.2.216] [PMID]

[13] Garcia.FE, Barraza-Pena CG, Wlodarczyk A, Alvear-Carrasco M, ReyesReyes A. Psychometric properties of the Brief-COPE for the evaluation of coping strategies in the Chilean population. Psicol Reflex Crítica. 2018; 31:22. [DOI:10.1186/s41155-018-0102-3] [PMID] [PMCID]

[14] Campos M, Iraurgu J, Paez D, Velasco C. Afrontamiento y regulaciónemocional de hechosestresantes: Un meta-análisis de 13 estudios (coping and emotional regulation of stressful events: A metaanalysis of 13 studies) Bull Psicol. 2004; 82:25-44. https://psycnet.apa. org/record/2005-14074-002
[15] García FE, Paez D, Cartes-Rovira G, Zurtia GC, Martel HN, Reyes AR. Religious coping, social support and subjective severity as predictors of posttraumatic growth in people affected by the earthquake in Chile on Religions. 2014; 5:1132-45. [DOI:10.3390/rel5041132]

[16] Schnider KR, Elhai JD, Gray MJ. Coping style use predicts posttraumatic stress and complicated grief symptom severity among college students reporting a traumatic loss. J Counsel Psychol. 2007; 54(3):344-50. [DOI:10.1037/0022-0167.54.3.344]

[17] Reich M, Costa-Ball CD, Remor E. Estudio de las propiedadesp sicometricas del brief COPE para una muestra de mujeresuruguayas (Psychometric properties of the brief COPE in a sample of uruguayan women). Avances Psicol Latinoam. 2016; 34(3):615-36. [DOI:10.12804/ apl34.3.2016.13]

[18] Carver CS, Scheier MF, Weintraub JK. Assessing coping strategies: A theoretically based approach. J Pers Soc Psychol. 1989; 56(2):267-83. [DOI:10.1037/0022-3514.56.2.267] [PMID]

[19] Deisinger JA, Cassisi JE, Whitaker SL. Relationships between copingstyle and PAI profiles in a community sample. J Clin Psychol. 1996 52(3):303-10. [DOI:10.1002/(SICI)1097-4679(199605)52:3<303::AIDJCLP7>3.0.CO;2-S

[20] Carver CS. You want to measure coping but your protocol's too long: Consider the brief. Int J Behav Med. 1997; 4(1):92-100. [DOI:10.1207/ s15327558ijbm0401_6] [PMID]

[21] Kasi PM, Naqvi HA, Afghan AK, Khawar T, Khan FH, Khan UZ, et al. Coping styles in patients with anxiety and depression. ISRN Psychiatry. 2012; 2012:128672. [DOI:10.5402/2012/128672] [PMID] [PMCID]

[22] Doron J, Trouillet R, Gana K, Boiché J, Neveu D, Ninot G. Examination of the hierarchical structure of the brief COPE in a French sample: Empirical and theoretical convergences. J Pers Assess. 2014; 96(5):567-75 [DOI:10 .1080/00223891.2014.886255] [PMID]

[23] Monzani D, Steca P, Greco A, D'Addario M, Cappelletti E, Pancanil L. The situational version of the brief COPE: Dimensionality and relationships with goal-related variables. Eur J Psychol. 2015; 11(2):295-310. [DOI:10.5964/ejop.v11i2.935] [PMID]

[24] Miyazaki Y, Bodenhorn N, Zalaquett C, Nig K. Factorial structure of brief COPE for international students attending U.S. colleges. Coll Stud J. 2008; 42(3):795-806. https://eric.ed.gov/?id=EJ816993

[25] García FE, Barraza-Peña CG, Wlodarczyk A, Alvear-Carrasco M, ReyesReyes A. Psychometric properties of the Brief-COPE for the evaluation of coping strategies in the Chilean population. Psicologia: Reflexão e Crítica. 2018; 31(1):22. [DOI:10.1186/s41155-018-0102-3]

[26] Ornelas Mejorada RE, Tufiño Tufiño MA, Vite Sierra A, Tena Guerrero O, Riveros Rosas A, Sánchez Sosa JJ. Afrontamiento pacientesen con cancer de mama en radioterapia: Análisis de la Escala COPE breve. [coping in breast cancer patients undergoing radiotheraphy: The brief COPE scale]. Psicologia Y Salud. 2013; 23(1):55-62. https://psycnet.apa.org/ record/2013-06377-006

[27] Knoll N, Rieckmann N, Schwarzer R. Coping as a mediator between personality and stress outcomes: A longitudinal study with cataract surgery patients. Eur J Personality. 2005; 19(3):229-47. [DOI:10.1002/ per.546]

[28] Zeynivandnezhad F, Rashed F, Kaooni A. Exploratoryfactor analysis for TPACK among Mathematics Teachers: Why, what and how. Anatol J Educ. 2019; 4(1):59-76. [DOI:10.29333/aje.2019.416a]

[29] Comrey AL, Lee HB. A first course in factor analysis. New York: Psychology Press; 2013. [DOI:10.4324/9781315827506] 
[30] Jalalinejad R, Yazdkhasti F, Abedi A. [Validity, reliability, and factor structure of corver, scheier and weinteraub's Coping Operations Preference Enquiry (COPE) in university of Isfahan students (Persian)]. Behav Cognit Sci J. 2014; 3(2):41-54. https://cbs.ui.ac.ir/article_17313. html?lang=en

[31] Wild D, Grove A, Martin M, Eremenco S, McElroy S, Verjee-Lorenz A, et al. Principles of good practice for the translation and cultural adaptation process for Patient-Reported Outcomes (PRO) measures: Report of the ISPOR task force for translation and cultural adaptation. Value Health. 2005; 8(2):94-104. [DOI:10.1111/j.1524-4733.2005.04054.x] [PMID]

[32] Polit DF, Beck CT, Owen SV. Is the CVI an acceptable indicator of contentvalidity? Appraisal and recommendations. Res Nurs Health. 2007; 30(4):459-67. [DOI:10.1002/nur.20199] [PMID]

[33] Meyers LS, Gamst G, Guarino AJ. Applied multivarite research: Design and interpretation. California: SAGE; 2006. https://books. google.com/books/about/Applied_Multivariate_Research. html?id=7e4npyN3BasC 
This Page Intentionally Left Blank 